NACIONAL

\title{
La seguridad nacional en el sistema normativo actual chileno: Análisis en relación con las recientes oportunidades en que ha sido invocada
}

\author{
National security in the current Chilean regulatory system: Analysis in relation \\ to recent opportunities in which it has been invoked
}

\section{Cynthia Alejandra Martinic Jara (D) Universidad de Chile}

\begin{abstract}
RESUMEN La seguridad nacional, al ser un concepto ambiguo y tradicionalmente indeterminado, se ha utilizado en algunas ocasiones como factor para imponer determinados intereses políticos a la población, para justificar violaciones a los derechos humanos y últimamente para frenar los cambios en curso. Este artículo desarrolla someramente el tratamiento doctrinal y que el sistema internacional de los derechos humanos y otros acuerdos internacionales le han dado al concepto de seguridad nacional y sus alcances. Se exponen además tres ejemplos recientes en Chile en que se ha invocado la seguridad nacional o la inteligencia estatal como su herramienta, con el objeto final de establecer la necesidad de que, con miras a una nueva constitución, se elabore y delimite un concepto de seguridad nacional que atienda las nuevas amenazas existentes, desmilitarice su tratamiento y control, y tenga como centro la dignidad humana.
\end{abstract}

PALABRAS CLAVE Seguridad nacional, inteligencia estatal, nueva constitución, estallido social, derechos humanos.

ABSTRACT National security, being an ambiguous and traditionally indeterminate concept, has been used on some occasions as a factor to impose certain political interests on the population, to justify human rights violations and lately with the aim of slowing down ongoing changes. This article briefly develops the doctrinal treatment that the international system of human rights and other international agreements have given to the concept of national security and its scope. It also exposes three recent examples in Chile in which national security or state intelligence (as a tool of the first) has been invoked, with the final objective of establishing, with a view to a new constitution, the need for a concept of national security that addresses the new existing threats, demilitarizes their treatment and control and has human dignity as its center. 
KEYWORDS National security, State intelligence, new constitution, social revolt, human rights.

\section{Introducción}

Durante el último año se ha empleado el argumento de la seguridad nacional con el objeto de justificar acciones del Gobierno o de sus brazos armados, tanto a propósito de la crisis social, económica y política que derivó en protestas ciudadanas desde el 18 de octubre de 2019, como por la pandemia del covid-19. Por ello, con miras a la redacción de una nueva constitución para Chile, este trabajo pretende analizar el tratamiento que nuestro sistema jurídico vigente le entrega al concepto de seguridad nacional y cómo han interpretado dicha normativa sus ejecutores en los contextos antes referidos.

Para llevar a cabo nuestro propósito, en un primer momento reseñaremos brevemente qué se ha entendido por seguridad nacional, tanto a nivel doctrinal como en la normativa del sistema internacional de los derechos humanos, para luego adentrarnos en el contenido de las distintas normas que componen el sistema normativo vigente chileno a su respecto. Luego, expondremos algunos ejemplos recientes en que la autoridad competente ha interpretado o empleado el concepto de seguridad nacional, con el objetivo de abordar ciertos criterios que el derecho internacional de los derechos humanos ha fijado al respecto. Por último, concluiremos el artículo indicando algunas orientaciones que debiese contemplar un nuevo tratamiento de la seguridad nacional en la nueva constitución.

\section{Sobre el concepto de seguridad nacional}

Primero, es preciso señalar que la seguridad nacional es parte de los fines del Estado. Los Estados fueron creados por las sociedades organizadas con el objeto de dejar atrás la autotutela, entendida como «un medio de solución del conflicto directo y unilateral mediante el que una parte no acepta subordinar su interés propio al ajeno y hace uso de la fuerza» (Delgado Castro, Palomo y Delgado, 2017: 269). Se le confirió entonces al Estado el ejercicio monopólico de la fuerza, por lo que tiene bajo su responsabilidad el garantizar la seguridad de los habitantes de la nación ${ }^{1}$ frente a eventuales conflictos o amenazas, sean internas o externas.

En un principio, la seguridad nacional se entendió en la lógica de protección del territorio de cada Estado ante la amenaza de guerra o invasión externa. Luego dio

1. Empleamos el vocablo nación bajo la antigua idea de «Estado-nación», ello sin perjuicio del efectivo reconocimiento de que las naciones pueden estar compuestas por estados federados, o que un Estado puede ser plurinacional. 
paso a relacionar la seguridad del Estado-nación con el resguardo de la soberanía, lo que incluyó la posibilidad de que existiesen conflictos o amenazas internas, además de las externas. Desde dicha óptica, la seguridad nacional está en manos de las Fuerzas Armadas de un país, se encuentra ligada al concepto de defensa nacional y se vincula con la actividad y capacidad coercitiva de los Estados. Como indica Gustavo de Greiff (2005: 151), «seguridad nacional es la ausencia de inseguridad para la soberanía nacional, tanto desde el punto de vista de amenazas provenientes del exterior, como las que tengan su origen al interior del territorio que abarca la nación».

Con el paso del tiempo y tras los horrores de las guerras mundiales, la Guerra Fría y la superación de las dictaduras militares en varios países latinoamericanos, ${ }^{2}$ han ocurrido dos fenómenos que podemos sindicar como causas de una evolución en el concepto de seguridad nacional. El primero dice relación con un enfoque del concepto de seguridad orientado hacia la protección de la persona y de la democracia como bienes jurídicos que debe cautelar la seguridad nacional - esto muy ligado con el desarrollo del derecho internacional de los derechos humanos-; el segundo, con la ampliación o incorporación de nuevas amenazas y de la mutación del carácter de los conflictos que pueden afectar al territorio, la soberanía, la democracia y a las personas.

Con la globalización y la mayor interdependencia internacional, se ha vislumbrado la necesidad de tener acuerdos respecto a eventuales amenazas que pueden incluso sufrir los países de manera colectiva. Es así como los Estados han definido, de manera particular o en concertación mediante acuerdos de derecho internacional, nuevas amenazas a la seguridad nacional, reconociendo entonces que dicho concepto tiene un carácter multidimensional y dinámico. En efecto, en la Declaración sobre Seguridad en las Américas de la OEA, de 2003, ${ }^{3}$ se identifican como eventuales amenazas a la seguridad nacional el terrorismo, el crimen organizado, el cambio climático, las pandemias, las emergencias naturales o causadas por el ser humano, el VIH, la pobreza extrema, el lavado de activos y los ciberataques, entre otros. ${ }^{4}$

En el caso de Chile, el Libro de la defensa nacional del Ministerio de Defensa Nacional, de 2017, indica que

2. Época en la que se acunó el concepto de la doctrina de la seguridad nacional, en que la seguridad nacional fue empleada como herramienta anticomunista y antisocialista. Para mayor análisis, véase Krsticevic y otros (2000).

3. «Declaración sobre seguridad en las Américas», Organización de los Estados Americanos, OEA/ Ser.K/XXXVIII, 17 y 28 de octubre de 2003, disponible en https://bit.ly/3jY43O9.

4. A este respecto, resulta también ilustrativa la Resolución CP/829 (1342/02), del Consejo Permanente de la Organización de los Estados Americanos, denominada «Adopción de los lineamientos sobre la elaboración de documentos sobre políticas y doctrinas nacionales de defensa», disponible en https://bit. ly/3yPECCv. 
la seguridad de la nación chilena debe entenderse como una condición que varía según las acciones que el Estado realice para hacer avanzar el país hacia los objetivos pretendidos y resguardar los intereses nacionales con la menor interferencia de riesgos, amenazas, problemas u otros obstáculos importantes (Ministerio de Defensa Nacional, 2017: 98).

Este además indica como objetivos de la defensa nacional:

Conservar la independencia y soberanía del país; mantener la integridad del territorio nacional; contribuir a la creación de condiciones de seguridad externa fundamentales para lograr el bien común de la nación; respaldar a la política exterior de Chile; contribuir al mantenimiento y promoción de la paz y la seguridad internacionales, de acuerdo con el interés nacional; contribuir al desarrollo nacional y cooperar al logro de otras capacidades del Estado; contribuir a la preservación de la institucionalidad de Chile como República democrática y al Estado de derecho; contribuir al mantenimiento del legado histórico y cultural de Chile; contribuir a las actividades que el Estado realiza con el propósito de fortalecer el compromiso ciudadano con la defensa; participar en el sistema de protección civil, contribuyendo a la respuesta oportuna ante situaciones de emergencia y catástrofe, en particular en acciones de protección a la población en condición de riesgo; contribuir, con la presencia de las Fuerzas Armadas en el territorio nacional, a la integración física de zonas fronterizas, aisladas y especiales, y al desarrollo social y económico de comunidades locales en dichas zonas (Ministerio de Defensa Nacional, 2017: 99).

En lo que respecta a la regulación de la seguridad nacional por parte de las normas que componen el sistema internacional de protección de los derechos humanos, si bien no encontramos una definición del concepto en dichos instrumentos, estos en términos generales vinculan la seguridad nacional a las nociones de guerra, peligro público o emergencia que amenace la vida de la nación, la seguridad de los Estados o su independencia. Así lo han definido el artículo 4.1. del Pacto de Derechos Civiles y Políticos, el artículo 15.1 del Convenio para la Protección de los Derechos Humanos y Libertades Fundamentales y el artículo 27.1 de la Convención Americana de Derechos Humanos.

Cabe agregar que la labor de inteligencia es una de las herramientas que los encargados de la seguridad nacional emplean como parte de las actividades encaminadas a obtener antecedentes necesarios para el resguardo de los intereses u objetivos establecidos para lograr la seguridad nacional. Es por ello por lo que existe una relación simbiótica entre el concepto de seguridad nacional que tenga un país y los propósitos y herramientas que emplee su sistema de inteligencia. Para Alejandro Martínez Serrano (2014: 244), «la inteligencia se refiere a la información importante para la formulación e instrumentación gubernamental a fin de mejorar sus intereses de [seguridad nacional] y enfrentar las amenazas de los adversarios potenciales contra 
aquellos intereses». A su vez, Leonardo Curzio Gutiérrez (2007: 107-108) postula que la inteligencia debe entenderse como necesaria para la supervivencia de un régimen democrático, requiriendo entonces de un acuerdo político sobre sus objetivos, prioridades, los órganos que la llevarán a cabo y su profesionalización.

Existe también una diferencia entre la seguridad nacional y la defensa nacional, por lo que no puede reducirse la primera a esta última. La defensa nacional empleada como sinónimo de seguridad nacional puede vulnerar los derechos humanos, pues su persecución puede llevar al uso de la fuerza militar.

Por último, la seguridad nacional debe diferenciarse del concepto de orden público, el que «incluye al normal funcionamiento de las instituciones básicas de la sociedad, la paz social y la convivencia pública» (Montalvo Abiol, 2010: 199), y que, al igual que la seguridad nacional, es un concepto ambiguo que al emplearse puede potencialmente limitar en sus efectos los derechos y libertades de las personas.

\section{La seguridad nacional en las normas chilenas}

El artículo 1, inciso final de la Constitución Política de la Republica señala como deber del Estado el resguardar la seguridad nacional.

Luego, el artículo 19, numeral 11, inciso segundo, indica que la Constitución asegura a todas las personas la libertad de enseñanza, prescribiendo que esta «no tiene otras limitaciones que las impuestas por la moral, las buenas costumbres, el orden público y la seguridad nacional». El artículo 19, numeral 16, inciso penúltimo, al regular el derecho a huelga, prohíbe la declaración de huelga de los funcionarios de servicios de utilidad pública o cuya paralización cause daño a la seguridad nacional. El artículo 19, numeral 21, establece el derecho de las personas a desarrollar cualquier actividad económica que no sea contraria a la moral, el orden público y la seguridad nacional. Por último, el artículo 19, numeral 24, que regula el derecho de propiedad, establece que las limitaciones u obligaciones al derecho de propiedad, o a cualquiera de sus atributos, deben derivar de su función social, entendiéndose por esta los intereses generales de la nación y la seguridad nacional.

Más adelante, el artículo 22, inciso segundo establece que «los chilenos tienen el deber fundamental de honrar a la patria, de defender su soberanía y contribuir a preservar la seguridad nacional». Por su parte, el artículo 32 sindica como atribuciones del presidente de la República: en el numeral 17, disponer de las fuerzas de aire, mar y tierra, organizarlas y distribuirlas de acuerdo con las necesidades de la seguridad nacional; el numeral 19 permite al presidente declarar la guerra, debiendo eso sí haber oído antes al Consejo de Seguridad Nacional (Cosena); y el numeral 20 autoriza al presidente a decretar pagos no autorizados para atender a necesidades impostergables derivadas de un grave daño o peligro para la seguridad nacional.

Luego, el artículo 101 consagra que las Fuerzas Armadas existen para la defensa de 
la patria y son esenciales para la seguridad nacional. Y en último término, la Constitución regula en los artículos 106 y 107 el Cosena, órgano que asesora al presidente en materias vinculadas a la seguridad nacional.

Expuesto lo anterior, es preciso indicar algunas normas atingentes a las disposiciones constitucionales antes referidas.

La Ley 18.948, orgánica constitucional de las Fuerzas Armadas, señala en su artículo 1 que las Fuerzas Armadas dependen del Ministerio de Defensa Nacional y «constituyen cuerpos armados que existen para la defensa de la patria, son esenciales para la seguridad nacional y garantizan el orden institucional de la República». El inciso segundo ahonda en que la consecución de los fines anteriores es permanente y descansa en un adecuado nivel de alistamiento del personal, del material y en el cumplimiento del juramento del servicio a la patria y defensa de sus valores fundamentales.

El DFL 2, de 2009, del Ministerio de Educación, permite en sus artículos 64, 74 y 81 el revocar el reconocimiento oficial de las universidades, institutos técnicos profesionales y centros de formación técnica, por realizar actividades contrarias a la moral, al orden público, a las buenas costumbres y a la seguridad nacional. Por otra parte, la Ley 18.840, orgánica constitucional del Banco Central, permite en su artículo 27 el financiamiento de créditos al Estado y a entidades públicas o privadas, en caso de guerra o peligro de ella.

El DFL 2, de 2001, del Ministerio de Hacienda, en su artículo 7 prohíbe el ingreso por zona francas de mercancías que atenten contra la seguridad nacional. Y el Decreto 1.094, de 1975, del Ministerio del Interior, que establece normas sobre extranjeros en Chile, prohíbe en su artículo 2 el ingreso al país a determinados extranjeros por interés o seguridad nacional. Esta prohibición se materializa por decreto supremo. Además, en su artículo 38 permite al Ministerio del Interior y Seguridad Pública revocar o denegar, por razones de orden público o seguridad nacional, la emisión de un documento de viaje a refugiados o asilados que requieran salir o reingresar a Chile.

La Ley 19.628, sobre Protección a la Vida Privada, establece en su artículo 15 que no podrá requerirse información o solicitar la cancelación o bloqueo de datos «cuando ello afecte la reserva o secreto establecidos en disposiciones legales o reglamentarias, la seguridad de la nación o el interés nacional». Añade en su artículo 16 que no se puede recurrir por el procedimiento especial que regula ley ante los juzgados civiles si la información se niega por motivos de seguridad nacional, por lo que debe en dicho caso deducirse una reclamación ante la Corte Suprema, la que solicitará informe y podrá recibir la causa a prueba.

El artículo 209 del Código Procesal Penal regula las diligencias investigativas de entrada y registro en lugares especiales, como los lugares religiosos, los edificios en que funcionare alguna autoridad pública o recintos militares, debiendo el fiscal en dichos casos previamente oficiarles. Establece luego que si «la diligencia implicare el 
examen de documentos reservados o de lugares en que se encontrare información o elementos de dicho carácter y cuyo conocimiento pudiere afectar la seguridad nacional», el encargado de la diligencia deberá oficiar al ministro respectivo para que este pueda oponerse. Si el fiscal persiste y cuenta con el apoyo del fiscal regional, podrá solicitar a la Corte Suprema que resuelva la controversia.

El Decreto 890, de 1975, del Ministerio del Interior, que refunda la Ley 12.927 sobre Seguridad Interior del Estado, tipifica delitos que se cometen en contra de la soberanía nacional, como los que ofendan gravemente el sentimiento patrio y la independencia política la nación, los que propiciaren la incorporación de todo o parte del territorio nacional a un Estado extranjero, los que presten ayuda a una potencia extranjera con el fin de desconocer el principio de autodeterminación del pueblo chileno o de someterse a su dominio político, entre otros. Luego, su artículo 4 tipifica los delitos contra la seguridad interior del Estado, como incitar o inducir a la subversión del orden público o a la revuelta, resistencia o derrocamiento del Gobierno constituido; los que inciten o induzcan a las Fuerzas Armadas o Policiales a la indisciplina o al desobedecimiento de las órdenes del Gobierno o sus superiores jerárquicos; los que concierten o faciliten reuniones destinadas a proponer el derrocamiento del Gobierno; los que inciten, induzcan, financien o ayuden a milicias privadas o semejantes, con el fin de sustituir la fuerza pública o atacarla; los militares o policías que no cumplan órdenes que imparta el Gobierno; los que propaguen doctrinas que tiendan a destruir por la violencia el orden social o la forma republicana y democrática de Gobierno; los que propaguen noticias falsas o tendenciosas destinadas a destruir el régimen de Gobierno, el orden constitucional, la seguridad del país, el régimen económico o el abastecimiento, entre otros.

Se encuentra también la Ley 19.974, sobre el Sistema de Inteligencia del Estado (SIE) y Crea la Agencia Nacional de Inteligencia. El fin del SIE según la Estrategia Nacional de Inteligencia 2019, aprobada mediante el Decreto 578, de 2019, del Ministerio del Interior y Seguridad Pública, «es contribuir a proteger la soberanía nacional y la integridad territorial, preservar el orden constitucional y formular apreciaciones de inteligencia útiles para la consecución de los objetivos nacionales». Dicho decreto además define como inteligencia de Estado a «aquella información procesada que permite proporcionar a las más altas autoridades la comprensión de los escenarios de interés, permitiéndoles minimizar los riesgos y hacer frente a las posibles amenazas $y$ oportunidades que se puedan manifestar y afecten los objetivos nacionales».

Como podemos notar, la seguridad nacional es empleada en nuestra legislación en términos amplios, ligados a la defensa nacional, de ciertos valores indeterminados, además de confundirse con el concepto de orden público. 


\section{Exposición de ejemplos recientes}

A continuación, se expondrán algunos ejemplos en los que se ha invocado la seguridad nacional en Chile, esto a propósito del «estallido social» y de la pandemia por covid-19.

En noviembre del 2019, el presidente de la República citó al Cosena para presentar una agenda de seguridad, atendido a los hechos de violencia ligados al estallido social. Este paquete de proyectos legislativos contenía, entre otras normas, las leyes denominadas por el propio Gobierno como «antibarricadas», «antiencapuchados» $y$ «antisaqueos», junto con la modernización de la Agencia Nacional de Inteligencia, de la Policía de Investigaciones y de Carabineros. ${ }^{5}$

El mismo año se supo de la incorporación de un miembro de Carabineros como infiltrado en una organización vecinal de la población Lo Hermida, de Peñalolén. Según la investigación del medio digital Ciper, dicho infiltrado, amparado en su anonimato y actuar por las instituciones de inteligencia estatal, habría incitado a los miembros de dicha organización a cometer delitos contra el orden público. ${ }^{6}$

Por último, a propósito de querellas penales interpuestas por los delitos de negativa o retardo de protección o servicio, denegación de servicio, homicidio omisivo y falsificación de instrumento público, denegación de auxilio, retardo o negativa de protección o servicios y homicidio imprudente, todo producto de los servicios que prestaron las entidades vinculadas a la protección de la salud y las decisiones de la autoridad para enfrentar los efectos de la pandemia de covid-19, la Fiscalía requirió la entrada y registro a dependencias del Ministerio de Salud para incautar información contenida en correos electrónicos, a lo cual se opuso su ministro por motivos de seguridad nacional. La Corte Suprema, que conoció de la controversia, ordenó después la exhibición parcial de los correos electrónicos. ${ }^{7}$

Estas son tres oportunidades muy disímiles en que la seguridad nacional ha sido interpretada por las autoridades de Gobierno sin desarrollar sus motivos para emplearla. En los primeros dos ejemplos podemos observar que se tergiversa la labor de mantención del orden público y se confunde con la seguridad nacional. En el último caso, se argumenta la seguridad nacional aun cuando la información que se buscaba recabar era útil para la protección de la salud pública amenazada por una pandemia.

Lo anterior da cuenta de la necesidad de que en la nueva constitución se delimite

5. Cabe precisar que durante el desarrollo de esta instancia, el contralor general de la República, miembro del Cosena, indicó que la convocatoria era improcedente, pues se basaba en hechos y propuestas relacionadas con el orden público y no con la seguridad nacional.

6. Nicolás Sepúlveda, «Carabinero infiltrado en Lo Hermida fue descubierto porque protagonizó programa de Canal 13 con su identidad real», Ciper, 15 de octubre de 2020, disponible en https://bit. ly/3AN4YHd.

7. Sentencia del caso Armendáriz con Paris, Corte Suprema, causa 112.505-2020, 15 de octubre de 2020. 
qué entenderemos por seguridad nacional, cómo podrá invocarla la autoridad, cuál es el procedimiento que deberá seguir y qué derechos podrá limitar o restringir.

\section{Tratamiento de la seguridad nacional a la luz del derecho internacional de los derechos humanos}

La regla general es que los derechos humanos no admiten restricciones. Por ende, de haber una restricción, esta debe provenir de una causa específica y extraordinaria. Luego, la posibilidad de restringir un derecho humano no deviene en un ejercicio arbitrario para los Estados, sino que esta facultad debe limitarse a la observancia del derecho internacional de los derechos humanos.

En el caso de la seguridad nacional, la restricción «legítima» de los derechos humanos se debe al cumplimiento de un objetivo legítimo por parte de los Estados relacionado con su seguridad y pervivencia, de conformidad a lo dispuesto en los artículos 15 y 16 de la Convención Americana de Derechos Humanos (CADH). Estas restricciones son de carácter particular, pues, como bien sabemos, existen derechos humanos que no admiten restricciones en lo absoluto, como aquellos que protegen a las personas en contra de la tortura, la esclavitud o la libertad de conciencia, y que además deben estar establecidas por ley y respetar el principio de proporcionalidad.

Las restricciones a los derechos humanos deben atender a la necesidad de preservar los principios y valores de una sociedad democrática. ${ }^{8}$ Por lo tanto, no sería legítimo el limitar los derechos humanos cuando esto socava a la democracia de un país.

Los Estados deben legislar sobre el procedimiento que emplearán si incurren en cláusulas de protección a la seguridad nacional. Por tanto, deben considerar que la restricción de derechos aplicada no puede en ningún caso comprometer el derecho mismo, conforme indican las reglas de interpretación contenidas en el artículo 29 de la CADH. Las medidas adoptadas por los Estados, además, no deben ser incompatibles con el respeto de los derechos humanos o de las personas, por lo que se debe aplicar un examen de necesidad y proporcionalidad de la medida que se adopte en relación con el objetivo que se pretende resguardar. ${ }^{9}$

8. Al respecto, resulta ilustrativo el considerando $148 .^{\circ}$ de la sentencia del caso Comunidad Indígena Yakye Axa con Paraguay, de la Corte Interamericana de Derecho Humanos, del 17 de junio de 2005, que señala: «La restricción que se haga al derecho a la propiedad privada de particulares pudiera ser necesaria para lograr el objetivo colectivo de preservar las identidades culturales en una sociedad democrática y pluralista en el sentido de la Convención Americana; y proporcional, si se hace el pago de una justa indemnización a los perjudicados, de conformidad con el artículo 21.2 de la Convención». Disponible en https://bit.ly/3hnDYpR.

9. «Cuadernillo de jurisprudencia de la Corte Interamericana de Derechos Humanos núm. 25: “Orden público y uso de la fuerza" ", Corte Interamericana de Derechos Humanos, 2020, disponible en https:// bit.ly/2TOVM49. 
Por último, en el caso del denominado «conflicto interno», la Corte Interamericana de Derechos Humanos ha señalado que «los Estados deben limitar al máximo el uso de las Fuerzas Armadas para el control de disturbios internos, puesto que el entrenamiento que reciben está dirigido a derrotar al enemigo, y no a la protección y control de civiles, entrenamiento que es propio de los entes policiales». ${ }^{10}$

\section{Hacia un nuevo trato de la seguridad nacional en una nueva constitución para Chile}

Atendido que bajo la doctrina de la seguridad nacional, el argumento fue empleado por los Estados para justificar violaciones de derechos humanos, ${ }^{11} \mathrm{y}$ considerando que la normativa chilena y los ejemplos antes expuestos - verificados además en contextos de limitaciones a derechos fundamentales generales- se han amparado en un concepto ambiguo de seguridad nacional, muy ligado a la noción de valores fundamentales (indeterminados, por lo demás, pero cuya protección se encuentra a cargo de la Fuerzas Armadas) y a su defensa militar, parece imperioso que en el proceso constituyente se debata ampliamente y se concuerde con los distintos sectores de la sociedad un término claro de seguridad nacional, en que se determinen los objetivos $\mathrm{e}$ intereses nacionales que protege, y se atienda a las nuevas amenazas existentes (que no necesariamente se enfrentan desde la vereda militar, como el cambio climático o la insuficiencia alimentaria), ligando el concepto a los de desarrollo y democracia. Procurando, además, que el tratamiento de la seguridad nacional que contemple la nueva constitución se restrinja y guíe expresa e indubitadamente por las normas internacionales de derechos humanos, y que, por tanto, la interpretación que de dicho concepto puedan hacer las autoridades y las medidas que puedan adoptar tengan como centro el respeto de la dignidad humana. Se debe, por lo demás, actualizar el concepto seguridad nacional a los derechos humanos, la libre determinación de los pueblos y el reconocimiento de la plurinacionalidad de los Estados.

\section{Referencias}

Curzio Gutiérrez, Leonardo (2007). La seguridad nacional en México y la relación con Estados Unidos. Ciudad de México: UNAM.

Delgado Castro, Jordi, Diego Palomo y Germán Delgado (2017). «Autotutela, solución adecuada del conflicto y reposesión: Revisión y propuesta». Revis-

10. Sentencia del caso Zambrano Vélez y otros con Ecuador, Corte Interamericana de Derechos Humanos, serie C núm. 166, fondo, reparaciones y costas, 4 de julio de 2007.

11. Al respecto, se recomienda revisar los casos seguidos y fallados por la Corte Interamericana de Derechos Humanos vinculados con la operación Condor: Ibsen Cárdenas e Ibsen Peña con Bolivia y Goiburu y otros con Paraguay. 
ta de Derecho (Universidad Católica del Norte), 24 (2): 265-289. DOI: 10.4067/ So718-97532017000200265.

GreIFF, Gustavo de (2005). «Terrorismo y seguridad nacional: El derecho internacional que hereda el siglo XXI». En Ricardo Méndez Silva (coordinador), Derecho y seguridad internacional: Memoria del Congreso Internacional de Culturas y Sistemas Jurídicos Comparados. Ciudad de México: UNAM.

Krsticevic, Viviana, José Miguel Vivanco, Juan E. Méndez y Drew Porter (2000). «Libertad de expresión y seguridad nacional en el sistema interamericano de protección de los derechos humanos». En Estudios básicos de derechos humanos (pp. 77-118). Tomo 10. San José: Instituto Interamericano de Derechos Humanos. Disponible en https://bit.ly/3xqKqCa.

Martínez Serrano, Alejandro (2014). "Tres momentos para entender la seguridad nacional de México». Revista de El Colegio de San Luis, 4 (7): 236-255. DOI: 10.21696/rcslo72014596.

Ministerio de Defensa Nacional de Chile (2017). Libro de defensa nacional. Santiago. Disponible en https://bit.ly/2SZF6GY.

Montalvo Abiol, Juan Carlos (2010). "Concepto de orden público en las democracias contemporáneas». Revista Jurídica Universidad Autónoma de Madrid, 22: 197-222. Disponible en https://bit.ly/3jSe4w7.

\section{Sobre la autora}

Cynthia Alejandra Martinic Jara es abogada y magíster en Derecho de la Universidad de Chile. Directora jurídica del estudio jurídico Arévalo y Paz Abogados. Su correo electrónico es cynthia.martinic@gmail.com. (D) https://orcid. org/0000-0002-7615-6111. 
El Anuario de Derechos Humanos es una publicación semestral de referencia y consulta en materia de derechos humanos y campos afines. Busca ser un espacio de discusión de los temas centrales en el ámbito nacional e internacional sobre derechos humanos. Es publicado desde 2005 por el Centro de Derechos Humanos de la Facultad de Derecho de la Universidad de Chile.

\author{
EDITORA \\ Claudia Iriarte Rivas \\ ciriarter@derecho.uchile.cl \\ SITIO WEB \\ anuariocdh.uchile.cl \\ CORREO ELECTRÓNICO \\ anuario-cdh@derecho.uchile.cl \\ LICENCIA DE ESTE ARTÍCULO \\ Creative Commons Atribución Compartir Igual 4.o Internacional
}

\author{
\% \\ La edición de textos, el diseño editorial \\ y la conversión a formatos electrónicos de este artículo \\ estuvieron a cargo de Tipográfica \\ (www.tipografica.io)
}

